\title{
ANALYSIS OF FINGER POSITION DURING TWO AND THREE-FINGERED GRASP: POSSIBLE IMPLICATIONS FOR TERMINAL DEVICE DESIGN
}

\author{
Murray E. Maitland ${ }^{1}$ and Molly Epstein, University of Washington, Department of \\ Rehabilitation Medicine, Seattle, Washington, USA
}

INTRODUCTION: Analysis of human hand kinesiology has been used to justify the design characteristics of terminal devices. The human hand is a complex mechanical structure consisting of 15 articulations controlled by over 30 muscles for the 5 fingers. It functions as a communication, sensory, grasp and manipulation structure. Separating essential hand kinesiology that is linked with each hand function might allow for the efficient development of terminal devices where there is one primary function such as grasping. It follows that to develop an effective grasping mechanism for a terminal device there should be a clear understanding of hand motions as they relate directly to the grasping function.

The conceptual framework used to characterize grasping functions will most likely influence the development of terminal devices. There is a growing body of literature that is refining the way we think of grasping activities, and may impact the design of terminal devices. For example, researchers have described the simplified coordination of hand motions during grasp as "synergies" or "virtual fingers", where there are predictable dependencies of one finger on another. Using conceptual approaches such as the "virtual fingers" concept will reduce the complexity of the description of the hand/object/task interaction.[2]

Recent studies indicate that only a small part of the hand's potential motion is used during grasp. Mason et al. [5] concluded that much of reach-to-grasp uses a base posture with small refinements in finger and thumb positions. In another study Baud-Bovy \& Soechting [1] found that when two fingers oppose the thumb, there is a predictable singular task that produced a balanced lever. Kamper et al [3] studied a variety of grasping tasks using 5 everyday objects. They found that the thumb, for example, used less than $5 \%$ of the available range. If one were to extrapolate these findings, prosthetic grasping mechanisms may only need a portion of the hand's total range of motion. Grasping patterns of the normal hand should be studied using a wide variety of objects to compare normal grasp patterns with prosthetic devices. The current study examined finger positions during twoand three-fingered grasp of a wide range of geometrical objects. The results from people with normal hands were compared to two terminal devices.

METHODS: Seven subjects participated in the study (2 male, 5 female). A 6-camera video motion analysis system (Qualisys, Gothenburg, Sweden) was used to collect marker locations in a calibrated volume approximately $1 \mathrm{~m}^{3}$. Fourteen reflective markers were taped to the interphalangeal joints, metacarpophalangeal joints and the carpals of the subjects (Figure 1). Seated subjects were asked to grasp objects, one at a time, using either two fingers or three fingers. The 9 objects were chosen based on standard geometric shapes, sizes and varied mass distribution properties. The smallest object was a $1.9 \mathrm{~cm}$ diameter sphere and the largest object was a 9.5 X 12.5 X $1.7 \mathrm{~cm}$ rectangular prism. The objects were painted brown to avoid inferring a particular use. Data were collected at 120

\footnotetext{
${ }^{1}$ The author has an intellectual property interest in a device discussed in this paper.
} 


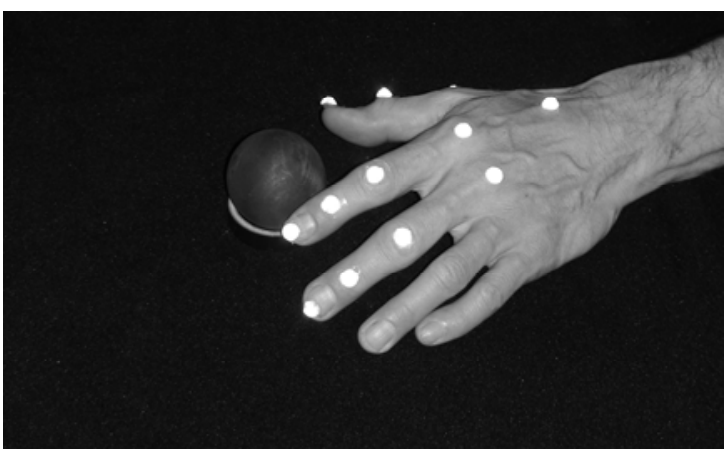

Figure 1. Grasping a medium-sized sphere

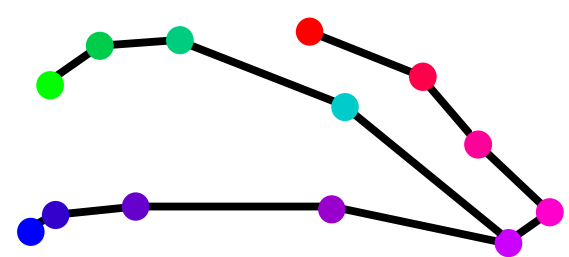

Figure 2. Stick model of grasping a mediumsized sphere frames/s and the markers' 3-dimensional coordinates were exported for further analysis using mathematical software.

The hand biomechanical model was modified from previous studies of grasp.[4] There were 11 rigid segments generated for the three dimensional motions of each of the finger articulations relative to the wrist (Figure 2). From the three dimensional coordinates of the markers, joint angles and contact angles were calculated.

Pearson product moment correlation coefficients (r) were used to determine the degree of association between distal to proximal joints as well as parallel joints between the fingers. A descriptive comparison was made between motion of the normal hand and two terminal devices (a hook and a modified hook).

RESULTS: Descriptive statistics of joint angles at the time of grasp are listed in Table 1. For two and three-fingered grasp using this specific set of objects, the thumb total range of motion was from $20^{\circ}$ to $43^{\circ}$ for $80 \%$ of grasps. In other words, the range of motion requirement for $80 \%$ of grasps in this sample was $23^{\circ}$. The largest variability of any of the finger joints was the index MCP joint (a range of $-44^{\circ}$ to $49^{\circ}$ ). There appeared to be a predisposition for the distal segments of the thumb and index finger to be angled rather than being parallel. The average angle between the index and thumb distal segments was about $30^{\circ}$.

\begin{tabular}{|c|c|c|c|c|c|}
\hline Table 1 & $\begin{array}{l}\text { Joint angle ave } \\
\text { Interphalangea }\end{array}$ & $\begin{array}{l}\text { ges, var } \\
\text { oint (IP) }\end{array}$ & $\begin{array}{l}\text { ability and ran } \\
\text { Metacarpoph } \\
\text { Standard } \\
\text { deviation }\end{array}$ & $\begin{array}{l}\text { e for all tria } \\
\text { angeal join } \\
\text { Minimum }\end{array}$ & $\begin{array}{l}\text { s and objects } \\
\text { (MCP) } \\
\text { Maximum }\end{array}$ \\
\hline \multirow[t]{3}{*}{ Thumb } & IP & 18 & 10 & 5 & 46 \\
\hline & МСР & 15 & 6 & 3 & 34 \\
\hline & $\begin{array}{l}\text { Total thumb } \\
\text { motion }\end{array}$ & 33 & 14 & 11 & 76 \\
\hline \multirow[t]{4}{*}{$\begin{array}{l}\text { Index } \\
\text { finger }\end{array}$} & Distal IP & 16 & 11 & 1 & 47 \\
\hline & Proximal IP & 24 & 9 & 5 & 39 \\
\hline & MCP & 18 & 28 & -44 & 49 \\
\hline & $\begin{array}{l}\text { Total index } \\
\text { motion }\end{array}$ & 72 & 18 & 38 & 112 \\
\hline \multirow[t]{4}{*}{$\begin{array}{l}\text { Long } \\
\text { finger }\end{array}$} & Distal IP & 19 & 14 & 3 & 49 \\
\hline & Proximal IP & 34 & 9 & 21 & 49 \\
\hline & МСР & 18 & 16 & -16 & 53 \\
\hline & $\begin{array}{l}\text { Total long } \\
\text { finger motion }\end{array}$ & 78 & 22 & 35 & 114 \\
\hline
\end{tabular}

Distal to proximal articulations in separate fingers (thumb, index and long fingers) had relatively low correlations $(r=-0.20$ to 0.40$)$. Thumb total range of motion had low correlation to index and long finger motion. Higher correlations were found with index finger and long finger parallel joints $(r=$ 0.41 to 0.65 ) and total 
From "MEC '08 Measuring Success in Upper Limb Prosthetics," Proceedings of the 2008 MyoElectric Controls/Powered Prosthetics Symposium, held in Fredericton, New Brunswick, Canada, August 13-15, 2008.

ranges of motion ( $\mathrm{r}=0.74)$.

The standard hook is a single hinge system (Figure 3). The hinge allowed for $70^{\circ}$ of motion for the distal grasp surface. The alternate terminal device mechanism included the proximal hinge of the standard hook and additional rotation through 4-bar linkages ${ }^{2}$ that allow contact surface motion of $90^{\circ}$ at each of the prosthetic "fingers" (Figure 4).

Grasp characteristics of the standard hook terminal device depend entirely on the single degree of freedom articulation relative to the size and surface geometry of the object. As a consequence, contact areas are relatively small.

The adapted hook device has a second degree of freedom at each contact surface mechanism that rotates in response to applied forces. The passive mechanisms become tangent to curved or flat object surfaces and can approximate the contact positions of the finger tips while grasping some objects.

DISCUSSION: The available sagittal plane range of motion of index and long finger distal phalanges relative to the metacarpals is approximately 320 degrees and the distal phalanx of the thumb can move 180 degrees relative to the $1^{\text {st }}$ metacarpal, but grasping patterns use only a small proportion of the total range. To develop an effective grasping terminal device, it might be possible to reproduce the necessary range of motion more easily than the potential range of motion.

One goal of a terminal device might be to obtain a similar contact position for the distal phalanx of the thumb, index and long fingers in relation to the objects. For example, the range of motion of a terminal device contact surface should be at least 90 degrees relative to the metacarpals while accommodating to the required aperture.

The single hinge of the hook device can create an aperture for grasp but there is no mechanism to bring the contact surfaces into a more normal finger to surface configuration. The standard hook device is unresponsive to change in forces at object interface. Friction coefficients are low, and in combination with the small surface contact areas and resultant forces on the object that push it away from the terminal device, grasping is less effective.

The adapted hook device has a second level of articulations that allow high-friction material to contact the object. Rotation of the 4-bar linkages allows improved contact geometry. Despite the potential for multiple contact points, the grasp patterns are not similar to the two or three finger grasp patterns of the normal hand. However, adaptations of mechanism may permit a more normal contact distribution.

While it is possible that only the biomimetic

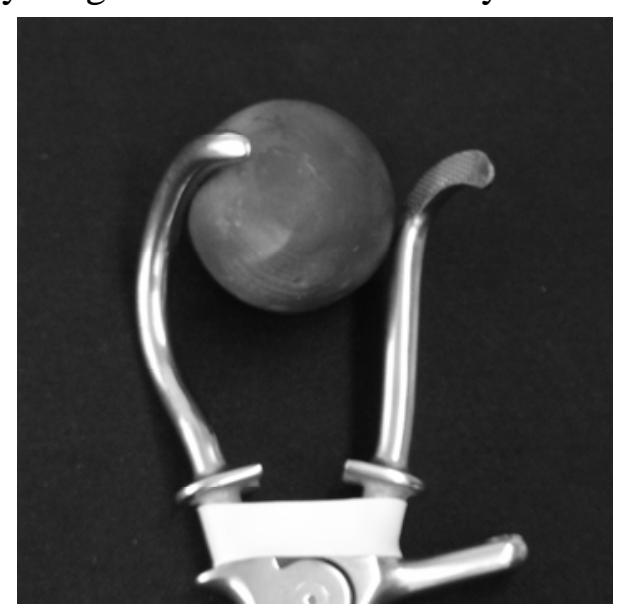

Figure 3 Hook terminal device grasping a sphere

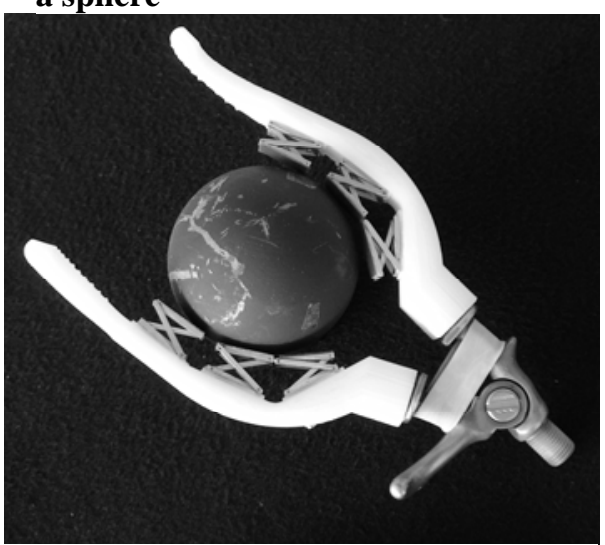

Figure 4 Adapted hook terminal device with 4-bar linkage components

\footnotetext{
${ }^{2}$ Patent pending
} 
approach can accomplish all possible grips, other approaches with simpler controls or mechanisms have not been explored thoroughly. In creating an artificial hand for grasping, the optimal configuration may differ from the anatomical configuration. Articular designs may lead to contact geometry that is similar to the normal hand.

CONCLUSIONS: Thumb position during two and three finger grasp of standard geometric objects was relatively consistent utilizing less than half of the available range of motion. Most grasps were accomplished within a $20^{\circ}$ range for the thumb. The index finger and long finger also used less than $50 \%$ of their available range of motion. Design criteria for prosthetic terminal devices for grasp should be derived from normal functional ranges of motion and finger positions used during the grasping process.

\section{Reference List}

[1] Baud-Bovy, G. \& Soechting, J. F., “Two virtual fingers in the control of the tripod grasp” J. Neurophys., 86, 604-615, 2001.

[2] Iberall, T., "The nature of human prehension: Three dextrous hands in one” IEEE International Conference on Robotics and Automation (Raleigh, NC) 396-401, 1987.

[3] Kamper, D. G., Cruz, E. G., \& Siegel, M. P., "Stereotypical fingertip trajectories during grasp” Journal of Neurophysiology, 90, 3702-3710, 2003.

[4] Lee, S.-W. \& Zhang, X., “Development and evaluation of an optimization-based model for power-grip posture prediction” J. Biomech., 38, 1591-1597, 2005.

[5] Mason, C. R. , Gomez, J. E. \& Ebner, T. J., “Hand synergies during reach-to-grasp” J. Neurophys., 86, 2896-2910, 2001. 